Results Drugs dispensed by ADSs represent $60 \%$ and $71.6 \%$ respectively of total medicines consumed in ICU and ED.

Four years after implantation:

- The quantity of drugs dispensed and drug stock has decreased in both units.

- The pharmacy department knows the type and amount of medicines to be found in each unit and in real time.

- The information it provides has helped to improve patient safety in relation to a better quality of prescription.

Since the implementation of $\mathrm{ADS}$, consumption has decreased compared to 2007:

\begin{tabular}{llll}
\hline Reduction in consumption in the Intensive & Care Unit from 2007 to: \\
\hline 2008 & 2009 & 2010 & \\
$-12.52 \%$ & $-16.86 \%$ & $-20.25 \%$ & $-40.65 \%$ \\
\hline Consume reduction in the emergency department from 2007 to: & \\
\hline 2008 & 2009 & 2010 & 2011 \\
$-1.49 \%$ & $-14.94 \%$ & $-29.18 \%$ & $-40.79 \%$ \\
\hline
\end{tabular}

Conclusions The implementation of $\mathrm{ADS}$ has meant an estimated saving of $938,330 €$.

The ADSs have increased drug control by the pharmacy department, have achieved a better rationalisation of resources and have improved efficiency in drugs use.

No conflict of interest.

\section{DSL-011 EFFICACY AND SAFETY OF EPOETIN ZETA IN DIALYSIS PATIENTS}

doi:10.1136/ejhpharm-2013-000276.254

'J Bajraktar, ${ }^{2} \mathrm{~B}$ Lazarova, ${ }^{3} \mathrm{E}$ Najdovska, ${ }^{2} \mathrm{~L}$ Mihajlova. ${ }^{1}$ Institute of nephrology, Hospital Pharmacy, Struga, FYROM; ${ }^{2}$ Clinical Hospital, Hospital Pharmacy, Stip, FYROM; ${ }^{3}$ Clinical Hospital, Hospital Pharmacy, Bitola, FYROM

Background Anaemia in chronic kidney disease (CKD) remains one of the predictable and modifiable non-traditional cardiovascular risk factors. Epoetin zeta, which is a biosimilar product, is used in the treatment of anaemia associated with chronic kidney disease.

Purpose This study was performed to evaluate the efficacy and safety of the biosimilar product epoetin zeta to maintain stable haemoglobin levels in dialysis patients.

Materials and Methods This study was conducted in 2 dialysis centres with 33 patients. Before the study 30 of the 33 patients were on various erythropoiesis-stimulating agents (ESA). After a run-in period of 2 months, all patients were switched to epoetin zeta and were followed for 6 months. The initial weekly doses as well as the frequency of use per week were kept constant (1-3 times/week). During the follow-up, haemoglobin levels, iron status, dialysis efficiency, body weight and adverse events were monitored at least once a month.

Results 33 patients were treated with biosimilar Epoetin zeta (27 men and 6 women); average age 59.1 (28-76) years; the frequency of used was 1-3 doses/week subcutaneously, over a period of 6 months. Dosing was to be adjusted to keep the Hb levels within 10.5-12 g/dl. Anaemia management and iron supplementation were at the discretion of the investigator and was to be in compliance with the current label. Throughout this study epoetin zeta was within the target range for Hb levels (10.5-11.5 g/dl $\pm 0.5 \mathrm{~g} / \mathrm{dl})$. The main AEs (adverse events) were in 1 patient hypotension (3\%), in 1 patient in-dialyzer clotting $(3 \%)$ and SAE (serious adverse event) was in 1 patient thrombosis of arteriovenous fistula (AVF) (3\%). No anti-epoetin antibodies and no clinical signs of pure red cell aplasia (PRCA) were observed in any patients on the study.

Conclusions Treatment of anaemia with Epoetin zeta was shown to be effective and safe. The mean Epoetin zeta doses remained stable in patients switched from all pre-study ESAs. The observed adverse events profile was in line with expectations for the study population.

No conflict of interest.

\section{DSL-012 EVALUATING THE STANDARDS OF HOSPITAL PHARMACIES IN THERAPEUTIC CENTRES AFFILIATED WITH OF KERMANSHAH UNIVERSITY OF MEDICAL SCIENCES, IRAN}

doi:10.1136/ejhpharm-2013-000276.255

'R Tahvilian, ${ }^{2 M R}$ Siahi Shadbad, ${ }^{3} \mathrm{H}$ Hamishehkar, 'V Aghababa Gharehbagh. ${ }^{1}$ Kermanshah University of Medical Sciences, Pharmaceutics, Kermanshah, Iran; ${ }^{2}$ Tabriz University of Medical Sciences, Pharmaceutics, Tabriz, Iran; ${ }^{3}$ Tabriz University of Medical Sciences, clinical pharmacy, Tabriz, Iran

Background Nowadays pharmaceutical care departments located in hospitals are amongst the important pillars of the healthcare system.

Purpose To evaluate the quality of hospital pharmacies affiliated to the Kermanshah University of Medical Sciences.

Materials and Methods In this cross-sectional study a validated questionnaire was used which enquired about all the necessary and standard requirements of an ideal hospital pharmacy. The questionnaire was filled in by the one of the researchers in all seventeen hospital pharmacies located in the teaching and non-teaching hospitals affiliated to the Kermanshah University of Medical Sciences. Data analysis was done using SPSS (version 17).

Results The results shows that in the hospitals observed, $24 \%$ of pharmacy environments, $25 \%$ of pharmacy store and storage conditions, $49 \%$ of storage procedures, $25 \%$ of drugs ordering and supplies, $73 \%$ of supplies reception (proper procedures followed for receiving supplies), 35\% of supplies reception (prompt action taken if deterioration of drugs received is suspected), $23.35 \%$ of drugs supplied to patients and finally $0 \%$ of stock cards used for inventory control met these standards in full. Several instances of incorrect processes of ordering, receiving, storing and delivering medicines to the patient were detected that have led to wasted money in hospitals and considerable decrease in the quality of medical services.

Conclusions Non-standard space allocation, incorrect ordering, receiving, storing processes and delivery of medicines to the patient were revealed by the questionnaire. These issues may reduce the efficiency and safety of pharmaceutical services and drug administration in hospitals.

No conflict of interest.

\section{DSL-013 EVALUATION OF THE LIMITS OF AUTOMATION AND IMPACT ON DRUG MANAGEMENT AT MOHAMMED V MILITARY TEACHING HOSPITAL PHARMACY, RABAT, MOROCCO}

doi:10.1136/ejhpharm-2013-000276.256

'W Enneffah, 'B Adouani, ${ }^{2} \mathrm{~S}$ Makram, ${ }^{3} \mathrm{~N}$ Cherkaoui, ${ }^{1} \mathrm{~A}$ Bennana. ${ }^{1}$ Mohammed V Military Teaching Hospital - Faculty of Medicine and Pharmacy, Clinical Pharmacy - Therapeutic Chemistry, Rabat, Morocco; ${ }^{2}$ Mohammed V Military Teaching Hospital - Faculty of Medicine and Pharmacy, Pharmacy - Pharmacology, Rabat, Morocco; ${ }^{3}$ Mohammed V Military Teaching Hospital - Faculty of Medicine and Pharmacy, Pharmacy - Galenic Pharmacy, Rabat, Morocco

Background Nowadays, hospitals tend to automate medicines management to increase quality, efficiency and safety of drug dispensing. At Mohammed V Military Teaching Hospital (MVMTH), a centralised Automated Drug Dispensing System (ADDS) was installed at the duty pharmacy. We expect this experience will be decentralised to all hospital services. 
Purpose To evaluate the impact of automation on medicines management at our duty pharmacy, and to determine its limits in order to improve them.

Materials and Methods We analysed the organisational aspects from the database of the ADDS deposited at the MVMTH duty pharmacy. The study lasted one year (2010). We also used a questionnaire completed at the end of the study period by the 12 Pharmacy Technicians (PharmTs) working at our hospital pharmacy ( 6 juniors with less than 5 years of professional experience and 6 seniors with more than 10 years of professional experience, all performing the same tasks during duty hours), in order to evaluate their view of automation.

\section{Results}

- 5444 transactions were accomplished ( $63 \%$ by juniors and $37 \%$ by seniors);

- injection forms were the most delivered (68\%) followed by oral forms (29\%);

- anti-inflammatories, analgesics and antispasmodics were the most required on duty hours (26\%) followed by antibiotics and antiviral drugs $(25 \%)$

- according to PharmTs:

- the main advantages were:

$\circ$ saving time in locating medicines $(\approx 83$ minutes saved per week, reallocated to other tasks): 8 PharmTs;

- limiting personal drug use: 5 PharmTs.

- the main constraints were:

- the irregular machine resupply (poorly done or not done at all) by the technician on duty whose job it is to replenish drugs consumed during the previous day: 10 PharmTs;

0 the reduced capacity for storing all medicines, especially refrigerated and oversized ones: 6 PharmTs.

Conclusions The automated drug dispensing system offers many advantages. However, there are still things to improve concerning machine resupply, storage capacity and storage conditions before decentralisation to hospital services.

No conflict of interest.

\section{DSL-014 FINANCIAL EVALUATION OF THE SURPLUS GENERATED BY THE DISPENSING OF SUNITINIB IN THE LOCAL HEALTH SERVICE OF REGGIO EMILIA}

doi:10.1136/ejhpharm-2013-000276.257

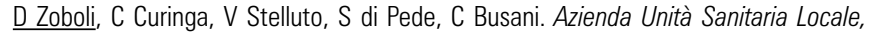
Pharmacy, Reggio Emilia, Italy

Background Oral cancer treatment has revolutionised the approach to the disease, as invasive procedures are no longer required, and patients can continue their daily life with practical and psychological benefits.

Purpose To cheque whether the packaging of Sutent (sunitinib), an oral cancer drug monitored by the Italian Medicines Agency (AIFA), dispensed by two District Pharmaceutical Units (UFD) of the Local Health Service (LHS) of Reggio Emilia, is suitable in terms of dosage units, to ensure coverage of the treatment plan prescribed to cancer patients. The marketing packaging authorised contains 30 capsules. Materials and Methods From September 2007 to September 2012, patients included in this study were dispensed Sutent at least once. We used the record of oncology drugs monitored closely by AIFA to obtain information about patients:

- number of prescriptions dispensed

- dose/day prescribed (e.g. $50 \mathrm{mg}$ );

- total dose prescribed (e.g. $1400 \mathrm{mg}$ );

- total dose dispensed (e.g. $1500 \mathrm{mg}$ );

The total dose of medicine prescribed, corresponding to one treatment cycle (i.e. 28 capsules) is less than the total dose of drug dispensed, corresponding to 30 capsules. For each cycle, there is a predictable surplus of 2 capsules.
Results More was paid than was necessary, for surplus Sutent, reported for 31 patients.

The overall cost of treatment provided in the study period was $€ 492,278$. The excess Sutent capsules represent $6.67 \%$ of the total cost of treatment, i.e. $€ 32,819$.

Conclusions In order to save money, it would help to dispense to the patient the exact number of dosage units required by the prescription.

This idea is supported by an analysis of the savings made for Xeloda. All patients received the exact number of dosage units in all UFD of the LHS of Reggio Emilia. From January 2010 to June 2012 savings were made by the Health Authority of $€ 57,602$.

No conflict of interest.

\section{DSL-015 FINANCIAL IMPACT OF ANTI-VEGF IN OPHTHALMOLOGY}

doi:10.1136/ejhpharm-2013-000276.258

A Pasquale, E Taormina, C La Seta. AOUP Paolo Glaccone, Farmacia, Palermo, Italy

Background The use of drugs with active ingredients produced through genetic engineering is often associated with oncology, rheumatology, dermatology and gastroenterology treatment although today there is wider use in cardiology (canakimumab) for certain products for the treatment of ocular pathologies, with particular reference to the retinal pathologies.

Currently the active principles used in ophthalmology are ranibizumab, pegaptanib and bevacizumab. The first two active ingredients are marketed in syringes ready for intravitreal use, but bevacizumab does not have a formulation different from that for use in oncology.

Ranibizumab is a fragment derived from the immunised antibody bevacizumab that exerts its anti-neogenic and vascular permeability-reducing actions by blocking VEGF (endothelial growth factors) with particular reference to isoforms VEGF165, VEGF121 and VEGF110. It is able to penetrate all the layers of the retina and enter the subretinal space.

Purpose To evaluate the economic impact of anti-VEGF drugs on the budget of the ophthalmology department and the average cost of treatment with ranibizumab considering a series of patients treated for age-related macular degeneration (AMD) at the Ophthalmology department in the Paolo Giaccone Hospital, Palermo.

Materials and Methods The consumption data were obtained from the accounting system of the integrated Polyclinic company, data on doses were obtained from a selection of patients who have had treatment from one to four years, and data were extracted from the AIFA monitoring log for ophthalmic medicines.

Results During the years 2007 to 2011 the share of the budget absorbed by anti-VEGF increased from $€ 58,375.1$ ( $45 \%$ of annual expenditure) to $246,592.71$ ( $84 \%$ of expenditure).

Given that the administration characteristics cannot be standardised we recorded the number of administrations for the patients treated.

8 patients that have been identified for a year's treatment received 3 to 4 administrations at an average cost per patient of Euro 4,023.25.

19 patients were treated for 2 years with average spending Euro 6,776 (4-9 doses) and a total cost 128,774 euros.

8 patients were treated for 3 years, average Euro 10,201.81 (6-13 doses) total expenditure Euro 81,614.5; Finally

5 patients were treated for 4 years, average Euro 13,334.2 (9-17 doses) total spending Euro 66,671.

It was possible to note that as the years of treatment increased the gap between of administrations widened.

Results In the near future the ageing population will increasingly request good treatment of $\mathrm{AMD}$. The latest ISTAT data indicate an increase in population over the age of 65 years (18.5\% of the population). Evaluating the incidence of AMD at $3.68 \%$ (EUREYE study) 